\title{
BioSpotlight
}

\section{Reflecting on Stem Cell Differentiation}

The ability to monitor stem cell differentiation is crucial in many developmental biology studies as well as when it comes to the prospect of using stem cells in regenerative medicine. This differentiation can be monitored by following the expression of specific cell surface markers; for example, the levels of stage-specific embryonic antigen-1 (SSEA 1) decrease when mouse embryonic stem (ES) and induced pluripotent stem (iPS) cells undergo differentiation. Typically, fluorescently-labeled antibodies are used for the immunocytochemical or flow cytometric detection of these markers, but such endpoint assays for antibodyantigen binding cannot measure binding reaction kinetics to determine the equilibrium dissociation constant $K_{d}$. In addition, fluorescent labeling can affect antibody strength and specificity. In this month's issue, W. Deng and X. Zhu, along with their colleagues at the U. of California, Davis (Davis, CA), demonstrate a label-free, real-time method for detecting the binding of unlabeled antibodies to stem cell surface markers. In their approach, stem cells are assayed on microarrays using oblique-incidence reflectivity difference (OI-RD) microscopy. Similar to surface plasmon resonance (SPR), OI-RD is an optical technique that can detect minute changes in the thickness or mass density of a substrateattached biomolecular layer due to the binding of solutionphase analytes. In OI-RD, these changes are observed as alterations in reflectivity, as measured by the ratio of the reflected electric field to the incident electric field. Compared to SPR scanning, OI-RD microscropy is more cost-effective since the biomolecular layers are attached to functionalized glass slides rather than a gold-coated substrate as required

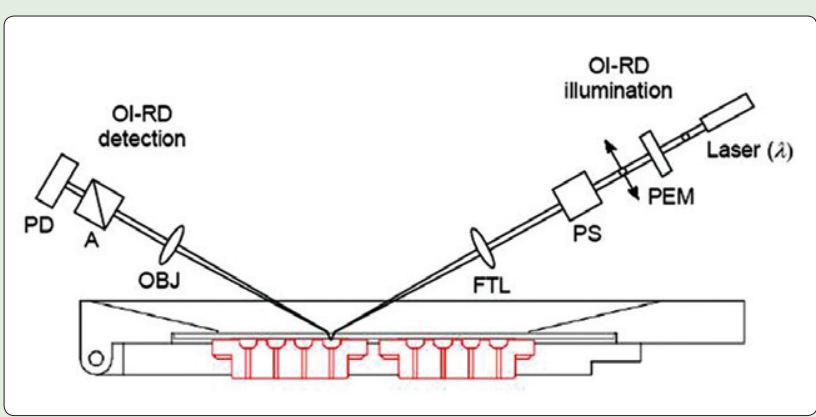

Schematic of the OI-RD scanning optical microscope consisting of illumination and detection optics and a sample cartridge holding a functionalized glass slide

in SPR, and its larger field-of-view allows coverage of up to 10,000 spots on a single microarray, resulting in a higher throughput. To validate the approach, mouse ES and iPS cells, either pluripotent or differentiated, were printed on aldehyde-coated glass slides and blocked with BSA. The resulting cell microarray was then reacted with anti-SSEA 1 antibody and observed in real time with an OI-RD microscope. Only stem cells, and not negative control cells, bound the antibody, and by fitting the real-time antibody association-dissociation data to a one-to-one Langmuir curve, $K_{d}$ 's for pluripotent vs. differentiated stem cells could be readily determined.

See "Label-free detection of surface markers on stem cells by oblique-incidence reflectivity difference microscopy" on page 381 .

\section{Fixing Dye Imaging}

While changes in cell shape and volume have been linked to essential biological processes including proliferation and locomotion, studying cells in three dimensions can be difficult, requiring highly specialized methods such as scanning electron or atomic force microscopy. Although some imaging methods such as differential interference contrast microscopy or confocal sectioning can provide a sense of the third dimension, most widely available techniques only provide accurate quantitative data for two dimensions. Transmission-through dye (TTD) imaging, developed by Model and colleagues at Kent State University (Kent, $\mathrm{OH})$, enables researchers to extract cell thickness information from a regular bright field image, generating data through which cell volume may be calculated. To use TTD imaging, living cells must first be covered with a non-toxic and strongly absorbing dye that cannot leak into cells. The cells are then imaged with near monochromatic illumination at the wavelength maximally absorbed by the dye. In regions where the cell body displaces the dye, more light will be transmitted, causing areas of increased cell thickness to appear brighter in the resulting images. Absolute values of cell thickness and volume can be calculated based on the amount of light transmitted through the sample at each point. TTD imaging is fast, has a high signal to noise ratio and can be performed using laser scanning or conventional microscopy with a bandpass filter. However, resolution is limited when using lower concentrations of dye, and increasing dye concentrations may interfere with cell function or block light penetration such that imaging thinner parts of a cell becomes difficult or impossible. Finding the right balance between the health of the cells during imaging, vertical resolution, and the ability to image at a maximum depth has been a challenge with this technique. Now, writing in this issue of BioTechniques, Model and colleagues describe an adaptation of
TTD microscopy to enable imaging of fixed cells, thus increasing vertical resolution by allowing use of high dye concentrations while also eliminating cell changes from occurring during observation. The authors discuss multiple fixation and dye combinations that can be used for TTD imaging, and present an optimal protocol for increasing vertical resolution using higher concentrations of the dye while balancing against the onset of fixation-induced artifacts.

See "Thickness profiling of formaldehyde-fixed cells by transmissionthrough-dye microscopy" on page 389 .

Selected and written by Patrick C.H. Lo, Ph.D. and Kristie Nybo, Ph.D. [⿷匚⿴囗十

BioTechniques 50:349 (June 2011)

doi 10.2144/000113678

To purchase reprints of this article, contact:

biotechniques@fosterprinting.com 